\title{
Presença de sintomas de fobia social, transtorno do pânico e ansiedade de separação em estudantes de 11 a 17 anos, em uma escola da rede pública de ensino de Salvador
}

\author{
Presence of symptoms of social phobia, panic and separation anxiety in students aged \\ 11 to 17 years from a school of th public system of Salvador.
}

Thais Prado', Curt Hemanny ${ }^{2}$, Irismar Reis de Oliveira ${ }^{3 *}$

${ }^{1}$ Psicóloga. Especialista em terapia familiar sistêmica e terapia cognitivo-comportamental para crianças e adolescentes. Mestranda do Programa de Pós-Graduação em Processos Interativos dos Órgãos e Sistemas, ICS/UFBA.; ${ }^{2}$ Psicólogo. Doutorando do Programa de Pós-Graduação em Processos Interativos dos Órgãos e Sistemas, ICS/UFBA.; ${ }^{3}$ Médico Psiquiatra. Doutor em Medicina e Saúde. Docente e professor titular aposentado do Departamento de Neurociências e Saúde Mental, Faculdade de Medicina/UFBA.

\begin{abstract}
Resumo
Introdução: crianças e adolescentes, não raro, apresentam sintomas de fobia social, transtorno do pânico e ansiedade de separação, que podem causar comprometimento nas habilidades sociais e dificultar as relações de forma incapacitante. Objetivo: Este estudo tem como objetivo verificar a presença de sintomas fóbicos sociais, transtorno de pânico e ansiedade de separação em adolescentes, com idade entre 11 e 17 anos, de uma escola pública da cidade de Salvador. Metodologia: este é um estudo observacional, transversal, que integra outro mais amplo, realizado em escola pública de Salvador, entre março e dezembro de 2015. A escala Revised Children's Anxiety and Depression foi aplicada em uma amostra de 674 alunos. Resultados: os alunos apresentaram sintomas de fobia social, transtorno do pânico e ansiedade de separação, em escala crescente, de acordo com a idade.

Palavra-chave: Fobia social. Pânico. Ansiedade. Adolescente.
\end{abstract}

\begin{abstract}
Introduction:children and adolescents often present symptoms of social phobia, panic disorder and separation anxiety disorder, which can cause social impairments and hinder relationships in a disabling way. Objective: this study aims to investigate the presence of social phobia, panic disorder and separation anxiety symptoms in adolescents aged between 11 and 17 years from a public school in the city of Salvador. Methodology: the present study is an observational cross-sectional research, which is part of a broader study conducted in a public school in Salvador, between March and December 2015. The Revised Children's Anxiety and Depression Scale (RCADS) was used in a sample of 674 students. Results: the students presented symptoms of social phobia, panic disorder and separation anxiety on an increasing scale proportional to age.

Keywords: Social phobia. Panic disorder. Anxiety disorder. Adolescents.
\end{abstract}

\section{INTRODUÇÃO}

A adolescência é considerada uma fase de transição entre a infância e a vida adulta. Segundo a Organização Mundial de Saúde (OMS), considerando o desenvolvimento cronológico, a adolescência compreende a fase entre 10 e 19 anos. No Brasil, segue-se a definição do Estatuto da Criança e do Adolescente (ECA), estabelecido na Lei 8.069, de 1990, que considera a faixa etária de 12 anos a 18 anos incompletos (artigo $2^{\circ}$ ) como o período que compreende a adolescência (GROLLI; WAGNER; DALBOSCO, 2017).

Por ser uma fase em que ocorrem muitas mudanças, os adolescentes são vulneráveis a diversos fatores de risco

Correspondente/Corresponding: *Irismar Reis de Oliveira-Instituto de Ciências da Saúde, Universidade Federal da Bahia - Endereço: Av. Reitor Miguel Calmon, s/n, Vale do Canela, Salvador - BA. CEP: 40110- 100 Tel: (71) 99981-9807-E-mail: Irismar.oliveira@uol.com.br para o surgimento de sintomas depressivos e ansiosos. Problemas emocionais, dificuldades familiares, problemas de saúde, experiências negativas, uso de drogas e álcool, além de comportamentos disfuncionais antissociais, podem desencadear sintomas que continuam, algumas vezes, na vida adulta (GROLLI; WAGNER; DALBOSCO, 2017; ROCHA; REIMÃO, 2010; SANTOS; SANTANA; SOUZA, 2020).

Apesar de a ansiedade ser considerada uma emoção natural e necessária ao ser humano, quando exagerada, pode trazer riscos para a saúde mental do indivíduo. Quando excessiva, mesmo na ausência de ameaça ou de forma desproporcional à ameaça, limitando a vida da pessoa, ela se torna patológica (STRÖHLE; GENSICHEN; DOMSCHKE, 2018). As causas do desenvolvimento de transtornos de ansiedade podem envolver interações complexas entre fatores biológicos, ambientais e indivi- 
duais, além de características individuais, como influência genética e temperamento. (STALLARD, 2010)

Um transtorno considerado frequente na infância e na adolescência é o transtorno de ansiedade social (TAS), também conhecido como fobia social (FS), que pode causar prejuízo nas habilidades sociais e dificultar os relacionamentos de forma incapacitante (COYLE et al., 2020; D'EL REY; PACINI; CHAVIRA, 2006; SANTOS;

UEHARA, 2019). Segundo estudos internacionais, a prevalência da FS em adolescentes situa-se entre 1,65\% a $7,2 \%$ (dependendo do instrumento utilizado). Em amostras clínicas, o diagnóstico de FS é mais comumente feito em jovens do sexo masculino. Porém, em estudos epidemiológicos, as mulheres são afetadas com maior frequência do que os homens (FERNANDES; TERRA, 2008; STRÖHLE; GENSICHEN; DOMSCHKE, 2018).

A FS é caracterizada como medo irracional, que provoca esquiva consciente do objeto ou situação temida. $\mathrm{O}$ indivíduo afetado apresenta sofrimento intenso na presença ou antecipação da entidade fóbica, prejudicando a sua capacidade de funcionamento como indivíduo. (COYLE et al., 2020; MARTINS; ALMEIDA; VIANA, 2014; SANTOS; UEHARA, 2019) Segundo o Manual Diagnóstico e Estatístico de Transtornos Mentais (DSM-V), em sua quinta edição, o critério diagnóstico para o FS consiste na presença de medo excessivo e persistente de situações nas quais a pessoa julga estar exposta à avaliação de terceiros; o indivíduo sente vergonha ou se comporta de maneira que pode prejudicar seu desempenho acadêmico, social e ocupacional. (AMERICAN PSYCHIATRIC ASSOCIATION, 2014; D'EL REY; PACINI; CHAVIRA, 2006; MARTINS; ALEMIDA; VIANA, 2014; TONGE et al., 2020) Em crianças e adolescentes, a dificuldade de compreensão do transtorno está relacionada ao desenvolvimento cognitivo e neurológico, ainda em formação (FERNANDES; TERRA, 2008).

Como a maior parte dos relacionamentos sociais ocorre na escola, os adolescentes que desenvolvem um quadro de FS tendem a apresentar comportamentos evitativos, tornando esse ambiente um lugar de grande desconforto e sofrimento. (AMERICAN PSYCHIATRIC ASSOCIATION, 2014; D'EL REY, 2006; SANTOS 2019, TONGE, 2020) O indivíduo pode apresentar sintomas como choro constante, irritabilidade, mutismo seletivo e recusa escolar (AMERICAN PSYCHIATRIC ASSOCIATION 2014).

A timidez transitória, retraimento social e temores à crítica social são características comuns dessa fase do desenvolvimento. Portanto, para ser considerada patológica, é necessário que sejam avaliados: o nível de sofrimento incapacitante e a duração do sintoma (AMERICAN PSYCHIATRIC ASSOCIATION, 2014; STRÖHLE; GENSICHEN; DOMSCHKE, 2018).

Vale ressaltar que, em grande parte dos casos, a FS se inicia na adolescência, podendo persistir na vida adulta. (MARTINS; ALMEIDA; VIANA, 2014; SANTOS; UEHARA, 2019; STRÖHLE; GENSICHEN; DOMSCHKE, 2018) Segundo o National
Comorbidity Survey (NCS), indivíduos com FS estão mais propensos ao desencadeamento de comorbidades, como transtorno depressivo maior, além de aumento do risco do desenvolvimento de transtornos por uso abusivo de álcool.

Em sua grande maioria, os quadros de FS são acompanhados por transtornos comórbidos, como: depressão maior, pânico, fobias específicas, transtorno de ansiedade generalizada, ansiedade de separação, abuso de substâncias, transtorno de personalidade evitativo, entre outros (SANTOS; UEHARA, 2019; STALLARD, 2010).

O transtorno do pânico (TP) tem como características ataques de ansiedade repentinos e inesperados, acompanhados de manifestações físicas (como dispneia, náuseas, palpitações, parestesia e diaforese) e mentais (temores relacionados à morte ou perda do controle, por exemplo) (AMERICAN PSYCHIATRIC ASSOCIATION, 2014; STRÖHLE; GENSICHEN; DOMSCHKE, 2018).

O transtorno de ansiedade de separação (AS) se caracteriza pela presença de sintomas de ansiedade de forma excessiva e persistente, após a separação de figura significativa do indivíduo. Apesar de a AS ocorrer, em grande parte na infância, $4,8 \%$ dos casos persistem ao longo da vida. Recentemente, a Organização Mundial de Saúde revelou dados da avaliação da Pesquisa Mundial de Saúde Mental, indicando que, de uma amostra de 38 993 adultos, em 18 países diferentes, a idade de início da AS era superior a 18 anos, em 43,1\% dos casos (STROHLE; GENSICHEN; DOMSCHKE, 2018).

Em sua maioria, os transtornos de ansiedade se iniciam na adolescência, principalmente nos casos de fobias específicas, como FS e TP. No caso da AS, em geral, o início dos sintomas acorre na infância. (STRÖHLE; GENSICHEN; DOMSCHKE, 2018) A maioria das crianças passa por uma fase transitória natural de aversão a estranhos, geralmente com início aos 8 ou 9 meses. Em 3\% das crianças, a AS se intensifica nos anos pré-escolares ou escolares. 0 tratamento é indicado quando há prejuízo no desenvolvimento normal da criança e ela deve ser tratada para que não haja prejuízo em vivências importantes, que devem ser experienciadas, proporcionando o desenvolvimento social (STRÖHLE; GENSICHEN; DOMSCHKE, 2018).

Estudos alertam sobre o comprometimento mental e funcional em crianças e adolescentes que apresentam sintomas de TAS, AS e TP, entre outros transtornos de ansiedade. Nesse sentido, o diagnóstico precoce se faz essencial, uma vez pode reduzir o prejuízo social nessas fases. A avaliação deve ocorrer de forma abrangente, envolvendo múltiplas fontes de informação, desde questionários de autorrelato, análise do histórico familiar até coleta de dados na escola (MARTINS; ALMEIDA; VIANA, 2014). Com relação ao tratamento, são disponíveis diversas opções, porém, é importante a realização de tratamentos combinados como psicoterapia (de preferência cognitivo- comportamental) e psicofarmacologia (WANG et al., 2018). 


\section{METODOLOGIA}

O presente estudo foi realizado em uma escola pública municipal da cidade de Salvador (BA), obedecendo às recomendações da Resolução CNS n. 466, de 12 de dezembro de 2012, referente ao desenvolvimento de pesquisa com seres humanos. Ele está inserido como parte de uma pesquisa mais ampla, intitulada Estudo da eficácia do treinamento cognitivo processual em grupo (TCP-G) na prevenção de transtorno de ansiedade e depressão em adolescentes de escolas públicas municipais e privadas de Salvador: um ensaio clínico randomizado, aprovada pelo Comitê de Ética em Pesquisa, da Maternidade Climério de Oliveira (Universidade Federal da Bahia), sob o número

966.202 de 27/02/2015, tendo como instituição proponente o Sanatório São Paulo, com número de processo CAAE: 42264315.0 .0000 .5543$.

O primeiro passo foi obter a autorização da Secretaria de Educação do Município de Salvador e da direção da escola municipal na qual os dados foram coletados. Em seguida, a partir de um encontro com os pais dos adolescentes, foram esclarecidos os objetivos do estudo e seu desenvolvimento, que ocorreu com os alunos em sala de aula. Os pais foram solicitados a assinar o Termo de Consentimento Livre e Esclarecido (TCLE) e os estudantes, a assinar o termo de assentimento.

Todos os procedimentos foram acordados antecipadamente e autorizados pela direção da escola. O primeiro encontro, realizado para a coleta de dados, ocorreu durante o turno da aula, onde os alunos receberam um caderno com um questionário de autorrelato, entregue pelos pesquisadores participantes da pesquisa maior e tiveram 40 minutos para preencher o instrumento e devolver para a equipe de pesquisa.

A amostra foi composta por 645 alunos, entre 12 e 17 anos, cursando o 70 e a 8 ano de uma escola pública da rede de ensino municipal de Salvador (BA). Os estudantes participaram de um treinamento de Terapia Cognitiva Processual em grupo (TCP-G) durante o semestre de 2016. Adotaram-se os seguintes critérios de inclusão: o aluno estare matriculado e cursando o ano letivo. Os alunos que não preencheram adequadamente os questionários e instrumentos psicométricos foram excluídos da amostra, bem como aqueles cujos pais ou responsáveis não assinaram o termo de consentimento.

Neste artigo, parte dos dados coletados foi discutida com base nos seguintes instrumentos: Escala Revisada de Ansiedade e Depressão Infantil (Revised Child Anxiety and Depression Scale-RCADS), para avaliar os sintomas de fobia social; e um questionário sociodemográfico para a caracterização da amostra, com informações relacionadas ao sexo, à idade e ao ano escolar.

Originada nos Estados Unidos, com autoria de Bruce Chorpita, a RCADS foi traduzida no Brasil. Ainda sem validação no país, pesquisas comprovaram que a versão para a língua portuguesa manteve as propriedades psicométricas do instrumento. A RCADS foi escolhida para ser utilizada na coleta de dados por se tratar de uma escala desenvolvida para avaliar a ansiedade e depressão. (CUMMINS; CAPORINO; KENDALL, 2014)

Ela se constitui em um questionário de autorrelato, composto por 47 itens, com subescalas correspondentes a transtorno de ansiedade de separação (AS), fobia social (FS), transtorno de ansiedade generalizada (TAG), transtorno de pânico (TP), transtorno obsessivo compulsivo (TOC) e transtorno depressivo maior (TDM). A soma das 5 subescalas de ansiedade formam uma escala de ansiedade total e a soma das 6 escalas, citadas anteriormente, formam uma Escala de Internalização Total. A RCADS possui, ainda, uma versão para os pais. (RCADS-P)

A referida escala solicita que os entrevistados avaliem a frequência com que cada item ocorre em suas vidas. Os itens são pontuados de 0 a 3 , respectivamente para "nunca", "às vezes", "frequentemente" e "sempre", tendo sido desenvolvida para avaliar síndromes clínicas em jovens. (CHORPITA; MOFFIT; GRAY, 2004)

No presente estudo, realizou-se uma análise descritiva dos dados, observando-se a presença de sintomas de FS, TP e AS, os quais foram comparados por sexo, idade e ano escolar. Para isso, foi utilizado o programa estatístico R. (RITTER; THEY; KONZEN, 2019; SILVA; DINIZ; BORTOLUZZI, 2009)

\section{RESULTADOS E DISCUSSÃO}

A Tabela 1 representa o perfil da amostra, segundo os dados coletados para sexo e idade, bem como pontuação da RCADS para sintomas de fobia social (FS), transtorno do Pânico (TP) e ansiedade de separação (AS).

Tabela 1 - Características sociodemográficas e clínicas da amostra

\begin{tabular}{lr} 
Idade M (DP) & $14,31(1,81)$ \\
\hline Sexo & \\
$\quad$ Feminino n (\%) & $351(52,2)$ \\
Masculino n (\%) & $322(47,8)$ \\
\hline RCADS-47 & $12,61(5,654)$ \\
Sintomas de Fobia Social M (DP) & $4,9(3,104)$ \\
Sintomas de ansiedade de separação M (DP) & $6,95(5,034)$ \\
Sintomas de Pânico M (DP)
\end{tabular}

Fonte: Dados da pesquisa.

Legenda: $M=$ Média; $D P=$ Desvio Padrão.

A Tabela 2 representa o perfil da amostra de acordo com sexo e pontuação da RCADS para sintomas de FS, TP e AS. 
Tabela 2 - Perfil por sexo e pontuação da RCADS

\begin{tabular}{lcr}
\hline RCADS - FS & N & M (DP) \\
Sexo & 341 & $14,01(5,596)$ \\
$\quad$ Feminino & 303 & $11,02(5,297)$ \\
$\quad$ Masculino & $\mathrm{N}$ & M (DP) \\
\hline RCADS - TP & 340 & $8,61(5,387)$ \\
Sexo & 303 & $5,08(3,839)$ \\
$\quad$ Feminino & & \\
$\quad$ Masculino & $\mathrm{N}$ & M (DP) \\
\hline RCADS-AS & 341 & $5,80(3,247)$ \\
Sexo & 303 & $3,89(2,596)$ \\
\hline Feminino & & \\
Masculino &
\end{tabular}

Fonte: Dados da pesquisa.

Legenda: $N=$ Frequência; $M=$ Média; $D P=$ Desvio Padrão; $R C A D S=$ Revised Children's Anxiety and Depression.

A Tabela 3 representa a correlação bivariada entre sintomas e o perfil da amostra, de acordo com sexo, idade e pontuação da escala RCADS para sintomas de FS, TP e AS.

Tabela 3 - Correlações bivariadas entre sintomas, idade e sexo

\begin{tabular}{l|c|c|c}
\multicolumn{1}{c|}{ Variáveis } & $\begin{array}{c}\text { Sintomas } \\
\text { de FS }\end{array}$ & $\begin{array}{c}\text { Sintomas } \\
\text { de TP }\end{array}$ & $\begin{array}{c}\text { Sintomas de } \\
\text { AS }\end{array}$ \\
\hline Idade & $0,081^{*}$ & 0,1 & 0,001 \\
\hline Sexo (Feminino) & $-0,267^{* *}$ & $-0,354^{* *}$ & $-0,306^{* *}$ \\
\hline Sintomas de FS & 1 & $0,580^{* *}$ & $0,521^{* *}$ \\
\hline Sintomas de TP & $0,580^{* *}$ & 1 & $0,570^{* *}$ \\
\hline Sintomas de AS & $0,521^{* *}$ & $0,570^{* *}$ & 1
\end{tabular}

Fonte: Dados da pesquisa.

Legenda: ${ }^{*} p \leq 0,05 ;{ }^{*} p \leq 0,0005 ; F S=$ Fobia Social, AS=Transtorno de Ansiedade de Separação, $T P=$ Transtorno de Pânico.

Na Tabela 3, observa-se que a idade se correlacionou positivamente com a presença de sintomas de FS ( $r$ de Pearson $=0,81 ; p<0,05)$, porém, com os sintomas de TP ( $r$ de Pearson $=0,10 ; p>0,05$ ) e AS ( $r$ de Pearson=0,001; $p>0,05)$, não ocorreu o mesmo. Com isso, observa-se que, quanto maior a idade, maior o score para FS, o que não ocorre com os sintomas de TP e de AS. Esses dados corroboram estudos que apontam a frequência de sintomas de ansiedade de separação com início, frequentemente, na infância (STRÖHLE; GENSICHEN; DOMSCHKE, 2018; WANG et al., 2017). Apesar da incidência de sintomas de AS se encontrarem, predominantemente, na infância, podem perdurar ao longo da adolescência e da vida adulta (BALDWIN; GORDON; ABELLE; PINI, 2016).

É possível verificar que, o sexo feminino está mais correlacionado positivamente, com a presença de sintomas de FS ( $r$ de Pearson=0,26; $p<0,005$ ), AS ( $r$ de Pearson $=0,3 ; p<0,005)$, e TP ( $r$ de Pearson=0,35; $p<0,0005)$.
Segundo Stallard (2010), os transtornos de ansiedade tendem a ser mais prevalentes em meninas, com maior idade. Em geral, meninos relatam menos fobias, TP e AS (STRÖHLE; GENSICHEN; DOMSCHKE, 2018).

A Tabela 3 apresenta as correlações bivariadas entre si e as correlações entre idade e sexo. Os sintomas de FS, TP e AS apresentam correlação positiva forte ( $r$ de Pearson $=0,580 * * ; p<0,0005 ; r$ de Pearson $=0,570 * *$; $p<0,005 ; r$ de Pearson $=0,521 * * ; p<0,005)$, significando que os sintomas de FS tendem a aumentar juntamente com os sintomas do TP e AS. Uma meta-análise envolvendo 20 estudos mostrou que crianças que apresentavam quadro de AS apresentaram três vezes mais risco de desenvolver o TP, do que a crianças sem AS (STRÖHLE; GENSICHEN; DOMSCHKE, 2018).

Por não se tratar de uma amostra clínica, não é possível verificar a prevalência FS, TP e AS. Verificamos na amostra uma presença considerável de sintomas, porém, suficientemente graves para se propor o diagnóstico desses transtornos.

\section{CONCLUSÃO}

O presente estudo proporciona aos profissionais a possibilidade de questionar sobre a necessidade do desenvolvimento de novas ações baseadas nas reais necessidades dos adolescentes no ambiente escolar. A presença de sintomas de ansiedade, mesmo sem gravidade, pode afetar o desempenho escolar e o desenvolvimento de habilidades sociais, necessárias para o manejo das relações ao longo da vida. Como visto anteriormente, os sintomas podem perdurar ao longo da adolescência e se estender à fase adulta. Mesmo quando se constata a presença de sintomas graves, a ansiedade pode ser negligenciada, por vergonha ou falta de informação. Nesse sentido, podemos destacar a importância do um diagnóstico precoce e tratamento multidisciplinar adequado, em parceria com a escola e familiares. A atenção à saúde mental vem ganhando importância, mas ainda necessita de estudos com mais evidências científicas.

\section{REFERÊNCIAS}

AMERICAN PSYCHIATRIC ASSOCIATION. DSM 5: manual diagnóstico e estatístico de transtornos mentais. 5. ed. Porto Alegre: Artmed, 2014.

BALDWIN, D. S.; GORDON, R.; ABELLI, M.; PINI, S. The separation of adult separation anxiety disorder. CNS Spectr., New York, v.4, n. 21, p. 289-294, Aug 2016. Disponível em: https://pubmed.ncbi.nlm.nih.gov/27503572/. Acesso em: 6 Sept. 2020. DOI: 10.1017/S1092852916000080.

CHORPITA, B. F.; MOFFIT, C. E.; GRAY, J. Psychometric properties of the Revised Child Anxiety and Depression Scale in a clinical sample. Behavior Research and Therapy, EUA, 2004. cap .43, p. 309-322. Disponível em: https://www.sciencedirect.com/science/article/pii/ S0005796704000695?via\%3Dihub. Acesso em: 5 Ago. 2020. DOI: 10.1016/j.brat.2004.02.004

COYLE, S. et al. School-based treatment for children and adolescents with social anxiety disorder. Social skills across the life span, theory, assessment, and intervention. EUA: Academic Press, 2020. cap 12, 
p. 237-254. Disponível em: https://www.sciencedirect.com/science/ article/pii/B9780128177525000123. Acesso em: 6 Nov. 2020. DOI: 10.1016/B978-0-12-817752-5.00012-3

CUMMINGS, C.M., CAPORINO, N.E.; KENDALL, P.C. Comorbidity of anxiety and depression in children and adolescents: 20 years after. Psychological Bulletin, Washington, v. 140, n. 3, p. 816-845, 2014. Disponível em: https://www.ncbi.nlm.nih.gov/pmc/articles/PMC4006306/. Acesso em: 01 set. 2020. DOI: 10.1037/a0034733.

D’EL REY, G.J.F.; PACINI, C. A.; CHAVIRA, D.J.F.. Fobia social em uma amostra de adolescentes. Estud. Psicol., Natal, v. 11, n. 1, p. 111-114, abr. 2006. Disponível em: http://www.scielo.br/scielo.php?script=sci_ arttext\&pid=S1413- 294X2006000100013\&lng=en\&nrm=iso. Acesso em: 9 nov. 2020. DOI:10.1590/S1413-294X2006000100013.

FERNANDES, G.C.; TERRA, M.B. Fobia social: estudo da prevalência em duas escolas em Porto Alegre. J. Bras. Psiquiatr, Rio de Janeiro, v. 57, n. 2, p. 122-126, 2008. Disponível em http://www.scielo.br/scielo. php?script=sci_arttext\&pid=S0047- 20852008000200007\&lng=en\&nrm =iso. Acesso em: 5 nov. 2020. DOI: 10.1590/S0047-20852008000200007.

GROLLI, V.; WAGNER, M.F.; DALBOSCO, S.N.P. Sintomas depressivos e de ansiedade em adolescentes do ensino médio. Revista de Psicologia da IMED, Passo Fundo, v. 9, n. 1, p. 87-103, nov. 2017. Disponível em: https://seer.imed.edu.br/index.php/revistapsico/article/view/2123. Acesso em: 10 nov. 2020. DOI:10.18256/2175-5027.2017.v9i1.2123

MARTINS, A.C.; ALMEIDA, J.P.; VIANA, V. Ansiedade social na infância e pré-adolescência: adaptação para o português de Portugal da SASC-R. Psicol. Reflex. Crit., Porto Alegre, v. 27, n. 2, p. 300-307, 2014 . Disponível em: http://www.scielo.br/scielo.php?script=sci_arttext\&pid=S010279722014000200300\&lng=en\&nrm=iso. Acesso em: 6 nov. 2020. DOI: 10.1590/1678-7153.201427210.

RITTER, M.N.; THEY, N. H.; KONZEN, E. Introdução ao software estatístico R. Imbé, RS: UFRGS, 2019. Disponível em: http://professor ufrgs.br/sites/ default/files/matiasritter/files/apostila_introducao ao_r_-_ritter_they_ and_konzen.pdf. Acesso em: 29 ago. 2020.

ROCHA, C.; REIMÃO, R. Associação entre sintomatologia depressiva e distúrbios do sono em adolescentes. In: VALLE, I.; MATTOS, M. (Orgs.).
Adolescência: as contradições da idade. 2. ed. Rio de Janeiro: Wak, 2010.

SANTOS, L.K.P.; SANTANA, C.C.; SOUZA, M.V.O. Ações para o fortalecimento da resiliência em adolescentes. Ciênc. Saúde Coletiva, Rio de Janeiro, v. 25, n. 10, p. 3933-3943, out. 2020. Disponível em: http://www.scielo.br/scielo.php?script=sci_arttext\&pid=S1413$81232020001003933 \& \mathrm{lng}=e n \& n r m=i s o$. Acesso em: 9 nov. 2020. DOI: 10.1590/1413-812320202510.22312018.

SANTOS, L.; UEHARA, E. Fobia social em adolescentes: repercussões acadêmicas. Revista de Psicologia da IMED, Passo Fundo, v. 8, n.2, p.172184. Dez. 2019. Disponível em: https://www.researchgate.net/publica tion/311786642FobiaSocialemadolescentesReperc ussoesAcademicas. Acesso em: 3 out. 2020. DOI:10.18256/2175-5027/psico- imed. v8n2p172-184

SILVA, B.F.; DINIZ, J.; BORTOLUZZI, M.A. Minicurso de estatística básica. Introdução ao software R. Santa Maria: Universidade Federal de Santa Maria, 2009.

STALLARD, P. Ansiedade: terapia cognitivo-comportamental para crianças e jovens. Porto Alegre: Artmed, 2010.

STRÖHLE, A.; GENSICHEN, J.; DOMSCHKE, K. The diagnosis and treatment of anxiety disorders. Deutsches Arzteblatt International, Deutschland, 2018. cap.155, v.37, p.611-620. Disponível em: https://www.ncbi. nlm.nih.gov/pmc/articles/PMC6206399/.Acesso em: 2 set. 2020. DOI:10.3238/arztebl.2018.0611.

TONGE, N. et al. Interpersonal problems in social anxiety disorder across different relational contexts. Journal of Anxiety Disorder, EUA, v. 75, p. 102275, 2020. Disponível em: http://www.sciencedirect.com/ science/article/pii/S088761852030089X. Acesso em: 3 out. 2020. DOI: 10.1016/j.janxdis.2020.102275.

WANG, Z. et al. Comparative effectiveness and safety of cognitive behavioral therapy and pharmacotherapy for childhood anxiety disorders: A systematic review and meta- analysis. JAMA Pediatr, EUA, v. 171, cap.11, p.1049-1056, 2017. Dosponível em: https://www.ncbi. nlm.nih.gov/pmc/articles/PMC5710373/. Acesso em: 3 out. 2020. DOI: 10.1001/jamapediatrics.2017.3036.

Submetido em: 08/12/2020

Aceito em: 14/12/2020 